\title{
2D Photocatalysts with Tuneable Supports for Enhanced Photocatalytic Water Splitting
}

Yiyang $\mathrm{Li}^{\mathrm{a}}$, Simson $\mathrm{Wu}^{\mathrm{a}}$, Jianwei Zheng ${ }^{\mathrm{a}}$, Yung-Kang Peng ${ }^{\mathrm{a}}$, Dharmalingam Prabhakaran ${ }^{\mathrm{b}}$, Robert A. Taylor ${ }^{\mathrm{b}}$ and Shik Chi Edman Tsang ${ }^{\text {a* }}$

${ }^{a}$ Wolfson Catalysis Centre, Department of Chemistry, University of Oxford, Oxford, OX1 3QR, United Kingdom

${ }^{\mathrm{b}}$ Clarendon Laboratory, Department of Physics, University of Oxford, OX1 3PU, United Kingdom

*Correspondence to: edman.tsang@chem.ox.ac.uk

\begin{abstract}
Sustainable hydrogen production is attracting increasing attention and visible-light-driven water splitting is considered as one of the most promising approaches for hydrogen evolution and solar energy storage. Different materials have been screened at mild conditions in the recent decades and 2-dimensional (2D) layered materials are considered as good candidates for the photocatalytic water splitting reaction. 2D single layer $\mathrm{MoS}_{2}$ has shown its potential in various catalytic systems, which has also been used in photocatalytic water splitting reaction recently. However, current studies of $\mathrm{MoS}_{2}$ monolayers give low intrinsic activity, preventing it from practical applications. This is attributed to the rapid recombination of the photo-excited charge carriers at room temperature, resulting in poor quantum efficiency $(\mathrm{QE})$. Herein, a state-of-the-art strategy to prolong the exciton lifetimes is reported, which is achieved by combining the $2 \mathrm{D} \mathrm{MoS}_{2}$ nanosheets with solid state polar-faceted supports. The charge separation process can be facilitated by the strong local polarisation introduced by the polar-faceted supports, and tuned by changing the supports with different surface polarities. Polar oxide surface is the exposure of oxygen-terminated high energetic facet, which is known to give a net dipole moment perpendicular to its surface. Such variation in the surface properties of the support to the above metal would lead to a difference in metal-support interaction(s). The resulted composite structures show great enhancement toward the visible-light-driven photocatalytic water splitting reaction, giving hydrogen and oxygen evolution in a stoichiometric 2:1 ratio at elevated temperatures from pure water. Photocatalytic performances are improved by the prolonged exciton lifetimes and exceptional hydrogen evolution activity of $2977 \mu \mathrm{mol} \mathrm{g}^{-1} \mathrm{~h}^{-1}$ with impressive quantum efficiencies are obtained over $\mathrm{Ru}$-doped $\mathrm{MoS}_{2}$ nanosheets on polar ceria support, which is among the best of the reported results of similar catalytic systems up to date. More excitingly, the linear relationship between the exciton lifetimes and strength of the local polarisation is also observed, indicating that the rational design of photocatalysts can be simply achieved via engineering their local polarisation by incorporation of polar-faceted materials.
\end{abstract}

Keywords: Photocatalytic water splitting; time-resolved techniques; single layer $\operatorname{MoS}_{2}$; polar-faceted materials 


\section{Introduction}

Two-dimensional (2D) materials such as graphene and transition metal dichalcogenides (TMDs), etc. have been proved to show a great potential in catalysis, energy conversion and many other fields in recent decades. Among the 2D materials, single molecular-layer $\mathrm{MoS}_{2}\left(\mathrm{SL}-\mathrm{MoS}_{2}\right)$ has attracted considerable attention for photocatalytic and electrochemical hydrogen evolution reaction (HER) due to its suitable band structure and low overpotential [1-5], and recent study also shows its potential in ammonia synthesis [6,7]. It has been reported that with the reduction of the thickness of $\mathrm{MoS}_{2}$, their optical, electronic and magnetic properties can change dramatically, leading to enhanced catalytic performances [8-10]. The absorption properties and band structures have been therefore well-studied [9], which depict that as $\mathrm{MoS}_{2}$ is exfoliated to monolayer, its bandgap is broadened, making its conduction band and valance band reach the proper positions for the hydrogen and oxygen evolution reactions, respectively [11]. Such properties make the SL-MoS ${ }_{2}$ as a promising candidate for solar-light-driven photocatalytic water splitting, which converts the fluctuated solar energy to stable chemical energy in the form of hydrogen. Theoretically, stoichiometric hydrogen and oxygen can be produced from pure water in the presence of the SL-MoS 2 based photocatalysts and sunlight. However, so far, the SL-MoS 2 are still commonly used as a substitute of noble metal like Pt in photocatalytic or electrocatalytic hydrogen evolution systems, but very few studies reported $\mathrm{MoS}_{2}$ alone as the photocatalyst. In addition, sacrificial reagents are frequently engaged in such photocatalytic water splitting systems to capture the photo-generated holes due to the fast intrinsic charge recombination process, leading to unwanted by-products and extra costs $[4,5,12]$. Therefore, strategies to facilitate the charge separation of this 2D material without using additive(s) are urgently needed.

Efforts have been made to suppress the charge recombination process of the 2D materials including SL$\mathrm{MoS}_{2}$, and approaches such as the heterojunction formation, defects engineering and introduction of internal electric fields have been developed [13-15]. Apparently, it is tremendously important to enhance the local polarisation within the photocatalyst particle to facilitate the separation of photoexcited electrons and holes $[15,16]$. Li et al. recently reported a Janus $\left(\mathrm{Cl}_{2}\right)-\left(\mathrm{Bi}_{12} \mathrm{O}_{17}\right)-\left(\mathrm{MoS}_{2}\right)$ bilayer junction structure as a photocatalyst which showed prolonged exciton lifetime due to the internal electric field, however, the use of sacrificial reagents is still inevitable [14]. DFT studies indicated that the edge-modification and heterojunction formation of the 2D materials can lead to enhanced photocatalytic water splitting performances, which, however, still lacks further experimental evidence $[17,18]$. Synthesis methods can also influence the physical and chemical properties of the SL-MoS 2 [19]. However, most of these strategies need very fine control and are only feasible in certain situations, which limit the use of these techniques. Moreover, oxygen and hydrogen cannot yet be produced stoichiometrically over SL-MoS 2 as hole-scavenger or another bandgap semiconductor is still required. Therefore, a facile and versatile strategy to introduce local polarisation and 
tune the charge separation will be greatly valuable. We have recently discovered that the photocatalytic overall water splitting activities of $\mathrm{N}$-doped $\mathrm{TiO}_{2}$ particles can be enhanced by mixing with polar-faceted $\mathrm{MgO}$ (111) support particles [20,21]. However, it is not easy to systematic vary the interfaces between the polar-faceted $\mathrm{MgO}$ (111) and the catalyst particle in proximity and see the correlation. Therefore, assembling the 2D SL-MoS 2 with polar-faceted materials is a more promising approach to tame the separation process of the photo-generated charge carriers, given the fact that the polar effect is quite localised and the structural flexibility of small SL-MoS 2 sheets to encapsulate on the support may lead to stronger interaction.

Herein, we propose a simple but versatile approach to modify the exciton lifetimes of 2D SL-MoS 2 by introducing a local electric field (LEF) through the use of polar-faceted materials. Polar-faceted metal oxides and layered double hydroxides (LDHs) were combined with the SL-MoS 2 to tune its separation process of the photo-generated charge carriers. The time-resolved photoluminescence (TRPL) technique was employed to investigate the extension of exciton lifetimes resulted from the local polarisation effect. The assembled materials were also utilised in the visible-light-driven overall water splitting reaction as photocatalysts. Remarkable promotion effect was achieved with the help of polar-faceted materials at the interfaces, which leads to much enhanced hydrogen evolution rate of $2977 \mu \mathrm{mol} \mathrm{g}^{-1} \mathrm{~h}^{-1}$ for SL-MoS ${ }_{2}$-based materials. In addition, impressive quantum efficiencies (QE) of $66.8 \%$ were obtained under visible light irradiation (437 $\mathrm{nm})$ at $270{ }^{\circ} \mathrm{C}$ without sacrificial additives. Subsequently, a systematic study was carried out and the interrelationships between photocatalytic activities, exciton lifetimes and polarities were derived quantitatively. Thus, in this work, the local polarisation effect is extensively demonstrated, which will contribute towards the rational design of the photocatalysts, not only for water splitting reaction, but may also be applicable for other photocatalytic processes.

\section{Results and discussion}

2D SL-MoS 2 was chemically exfoliated by lithium intercalation method, followed by different metal doping via hydrothermal process (Fig. 1A). The morphologies of the SL-MoS 2 were initially studied by transmission electron microscopy (TEM) and compared with the bulk form, of which the images are shown in Fig. 1B and Fig. S1. Given that bulk $\mathrm{MoS}_{2}$ mainly exhibits large particle of extensive layered-structures which is in accordance with the observations in literature [22,23], it is exciting to see the TEM images of the exfoliated SL-MoS $S_{2}$ which illustrate a thin sheet-like morphology. Atomic force microscopy (AFM) was subsequently engaged to confirm that the thickness of individual layers is about $0.6-0.7 \mathrm{~nm}$, which is in good accordance with the ca. $0.65 \mathrm{~nm}$ of the S-Mo-S building block (Fig. S2). The single layer morphology was also revealed by the high-angle annular dark-field scanning transmission electron microscopy (HAADF-STEM) image of 
the SL-MoS 2 , as shown in Fig. 1C. X-ray diffraction (XRD) was carried out before and after the exfoliation, and the patterns sharply decreased and disappeared after the exfoliation, showing nearly a flat line, implying the successful synthesis of mostly single layer nanosheets (Fig. S3). Different metals were then attached onto the single layer nanosheets via the identical hydrothermal process after the exfoliation, where a metal loading of ca. $1.8 \%$ was confirmed by ICP analysis [10]. XRD patterns of the metal-doped SL-MoS 2 are given in Fig. S3, which exhibit almost straight lines for all the samples. Enlarged XRD patterns at the range of 10$20^{\circ}$ are also shown, in which no obvious peaks of metal NPs can be observed and the original peak of bulk $\mathrm{MoS}_{2}$ at $2 \theta$ of $14.2^{\circ}$ is severely reduced for each pattern, suggesting the SL-MoS $\mathrm{S}_{2}$ kept as ultrathin layers and no apparent re-stacking took place even after the hydrothermal process. The HAADF-STEM images of a typical metal-doped SL-MoS 2 are shown in Fig. 1D and Fig. S4, implying single atoms are attached onto the single layer nanosheets and overlap the Mo and S positions. EPR results were obtained and shown in Fig. S5, as expected, SL-MoS 2 showed a strong EPR signal at around $\mathrm{g}=2.00$, which is originated from the sulphur vacancies, while bulk $\mathrm{MoS}_{2}$ remained silent on EPR measurements. EPR also showed that after the metal doping, the signal decreased dramatically compared with that observed in pure SL-MoS ${ }_{2}$, which suggested that the metal ions are favourably adsorbed and anchored on the sulphur vacancy sites. Timeresolved photoluminescence (TRPL) spectroscopy suggested that the exciton lifetime of the SL-MoS 2 is largely prolonged compared with its bulk counterpart, presumably due to the change of the band structures and the introduction of defects during the chemical exfoliation $[10,24]$.

Initial evaluation of the photocatalytic water splitting performance was carried out using the conditions in our previous study [20] and more details can be found in the Experimental Section. As shown in Table S1, with visible light excitation, the bulk $\mathrm{MoS}_{2}$ shows no activity toward this water splitting reaction at $270^{\circ} \mathrm{C}$, while SL- $\mathrm{MoS}_{2}$ exhibits a hydrogen evolution rate of $157 \mu \mathrm{mol} \mathrm{g}{ }^{-1} \mathrm{~h}^{-1}$. Controlled experiments that carried out in dark or in the absence of catalysts also showed no activity, confirming the hydrogen production is due to the photocatalytic water splitting over $\mathrm{MoS}_{2}$-based photocatalysts. Photocatalytic activities are largely enhanced after the metal doping, among which Ru-doped SL-MoS $2\left(\mathrm{Ru}: \mathrm{SL}-\mathrm{MoS}_{2}\right)$ shows the best hydrogen production ability, depicting a hydrogen evolution rate of $821 \mu \mathrm{mol} \mathrm{g}^{-1} \mathrm{~h}^{-1}$ (Table 1), presumably due to its similar hydrogen adsorption energy as Pt with low overpotential of hydrogen reduction [25]; meanwhile the inexpensive Fe-doped SL-MoS $2\left(\mathrm{Fe}: \mathrm{SL}-\mathrm{MoS}_{2}\right)$ also gives a promising activity of $615 \mu \mathrm{mol} \mathrm{g}^{-1} \mathrm{~h}^{-1}$ (as shown in Table S1 and Fig. S6). The following study was focused on the Ru:SL-MoS 2 , which shows the highest activity among all the metals studied here. As can be observed from the aforementioned characterisations including XRD, EPR, etc. (Fig. S3 and S5), the Ru:SL-MoS 2 maintains mainly the monolayer morphology after metal doping via hydrothermal method, and the S-vacancies are substantially decreased due to the anchoring of Ru atoms. HAADF-STEM image also indicates the dispersion of Ru atoms on the SL-MoS ${ }_{2}$, 
occupying Mo atop sites as well as the S-vacancy sites without extensive formation large metal nanoparticles or clusters (Fig. 1E). More importantly, to precisely investigate the local atomic and electronic structures of the Ru:SL-MoS 2 sample, X-ray absorption near edge structure (XANES) and extended X-ray absorption fine structure (EXAFS) measurements were conducted, which are shown in the Fig. 1F and 1G. Clearly, Fig. 1F shows the XANES curves at Ru K-edge of Ru:SL-MoS 2 sample and the Ru foil as reference. It is found that the $\mathrm{Ru}: \mathrm{SL}-\mathrm{MoS}_{2}$ shares nearly the same absorption edge as the $\mathrm{Ru}$ foil reference, indicating that the $\mathrm{Ru}$ in the sample has an oxidation state of zero. Fourier transformed (FT) EXAFS analysis was then performed at $\mathrm{Ru} \mathrm{K}$-edge to further investigate the local interaction of the doped Ru atoms (Fig. 1G). In the FT-EXAFS spectra of Ru K-edge, no peak related to Ru-Ru scattering at $2.42 \AA$ (before phase shift) could be observed, indicating the absence of $\mathrm{Ru}-\mathrm{Ru}$ bond in the $\mathrm{Ru}: \mathrm{SL}-\mathrm{MoS}_{2}$. Instead, the peak at $1.98 \pm 0.03 \AA$ was observed for $\mathrm{Ru}: \mathrm{SL}-\mathrm{MoS}_{2}$ which is attributed to the contribution of Ru-S scattering. Moreover, the coordination number was derived by fitting the EXAFS curves, showing a value of $2.8 \pm 0.3$ with an R-factor of $3.5 \%$, which is in accordance with our expectation that the doped $\mathrm{Ru}$ atoms are anchored in the S sites with each $\mathrm{Ru}$ is surrounded by 3 nearest sulphur atoms. More details of EXAFS fittings can be found in Fig. S7
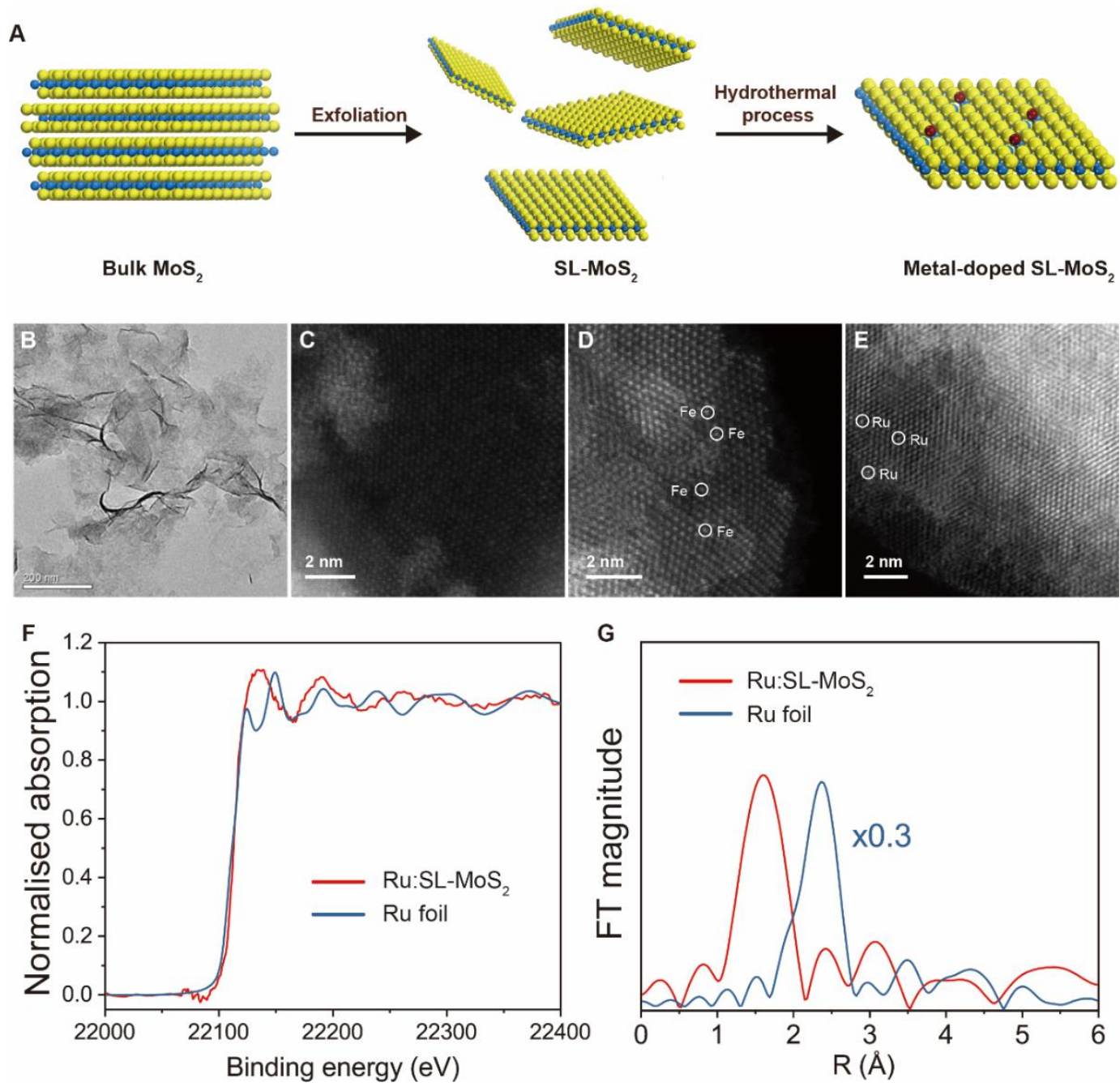
Figure 1. Synthesis and characterisations of the metal-doped SL-MoS $\mathbf{S}_{2}$ (A) Synthesis of the SL-MoS via chemical exfoliation and metal doped $\mathrm{SL}-\mathrm{MoS}_{2}$ via hydrothermal process (yellow: sulphur; blue: molybdenum; brown: doped metal atom); (B) conventional TEM image of the SL-MoS 2 ; HAADF-STEM image of (C) SL-MoS 2 ,(D) Fe:SL-MoS 2 and (E) Ru:SL-MoS 2 . Scales and doped metal atoms are labelled in the images; (F) XANES and (G) FT-EXAFS spectra of Ru:SL-MoS 2 for Ru K-edge.

Subsequently, the study of local polarisation effect was carried out by combining the Ru:SL-MoS 2 with various polar-faceted metal oxide supports, including $\mathrm{CeO}_{2}$ (100) nanocubes (NCs), $\mathrm{MgO}$ (111) and $\mathrm{ZnO}$ (0001) nanoplates (NPs), and their non-polar counterparts $\mathrm{CeO}_{2}$ nanospheres (NSs), $\mathrm{MgO}$ (100) and $\mathrm{ZnO}$ nanorods (NRs) were included for comparison. These polar surfaces display alternative layers of anions and cations hence the top surfaces will cover with non-cancellable polarity. Microscopic images of the polar and non-polar metal oxide supports are displayed in Figs. S8-10. For example, distinctive layers of $\mathrm{Ce}^{4+}$ and $\mathrm{O}^{2-}$ layers in (100) terminal facets can be directly visualised and indexed from Fig. S8. Such charge separation in alternative layers can generate strong surface polarity. Morphologies of $\mathrm{ZnO}$ nanoplates and nanorods are shown in Fig. S9, while those of $\mathrm{MgO}$ (111) and (100) are in Fig. S10, all of which indicate the successful engineering of the dominantly exposed facets. More detailed structural studies have also been reported elsewhere [26,27].The unique surface polarity of the polar oxide arrangement was further confirmed by the solid-state nuclear magnetic resonance (NMR) (Fig. S11). The probe-assisted ${ }^{31} \mathrm{P}$ NMR results clearly showed the different ${ }^{31} \mathrm{P}$ chemical shift of the polar-faceted metal oxides compared with their non-polar counterparts. More characterisations were then carried out to exclude the influence of the structural differences. XRD was carried out to investigate the structural information of the polar and non-polar metal oxides. It is clearly shown in Fig. S12 that the XRD patterns barely change with different dominant facet exposure. Taking $\mathrm{CeO}_{2}$ as an example, the nanocubes and nanospheres share almost the identical XRD patterns as can be seen in Fig. S12A, with the nanocubes exhibiting slightly stronger signals than the nanospheres. Such similarities also occur in the cases of $\mathrm{MgO}$ and $\mathrm{ZnO}$ samples (Figs. S12B and S12C). EPR analysis was then performed on the polar-faceted and non-polar oxides, which clearly indicates that $\mathrm{MgO}$ (111) and ZnO NPs show no obviously difference compared with their non-polar counterparts, while $\mathrm{CeO}_{2} \mathrm{NCs}$ shows higher signal intensity which is attributed to the formation of $\mathrm{Ce}^{3+}$ at the surface $[28,29]$, as shown in Fig. S13. As can be seen in the TEM images of a typical assembled photocatalyst, Ru:SL$\mathrm{MoS}_{2} / \mathrm{CeO}_{2}$ NCs (Fig. S14A and S14B), the SL-MoS 2 monolayers are well dispersed on the surface of the $\mathrm{CeO}_{2}$ nanocubes, which is further confirmed by the energy-dispersive spectroscopy (EDS) mapping (Fig. S14C and S14D). The photocatalytic water splitting activities of the metal oxide supports were also tested under the same conditions and the result show no hydrogen production (as shown in Table S1). 
The charge separation process of the assembled materials was investigated by the TRPL spectroscopy. Interestingly, the exciton lifetimes of the Ru:SL-MoS 2 were much prolonged, showing a more than 4-fold improvement from $1.02 \mathrm{~ns}$ to $4.53 \mathrm{~ns}$ after mixed with $\mathrm{CeO}_{2} \mathrm{NCs}$, and for the assembly with the $\mathrm{MgO}$ (111) and $\mathrm{ZnO}$ NPs, thus, the exciton lifetimes as shown in Fig. 2 are increased to 3.10 ns and 1.32 ns, respectively. However, those that combined with the non-polar metal oxides give no obvious change, as can be seen in Fig. 2A, Table 1 and Table S2. More direct comparison of the TRPL spectra between Ru:SL-MoS 2 , Ru:SL$\mathrm{MoS}_{2} / \mathrm{CeO}_{2} \mathrm{NCs}$ and $\mathrm{Ru}: \mathrm{SL}-\mathrm{MoS}_{2} / \mathrm{CeO}_{2} \mathrm{NSs}$ is also illustrated in the Fig. S15. Subsequently, the photocatalytic water splitting performance of these assembled materials were evaluated. Excitingly, the photocatalytic activity of Ru:SL-MoS ${ }_{2}$ was greatly enhanced after assembled to the polar-faceted oxides. As seen in Fig. 2C, the Ru:SL-MoS $/ \mathrm{CeO}_{2}$ NCs shows a high hydrogen evolution rate of $2977 \mu \mathrm{mol} \mathrm{g}^{-1} \mathrm{~h}^{-1}$ under visible light illumination, while those of Ru:SL-MoS $/ \mathrm{MgO}(111)$ and $\mathrm{Ru}: \mathrm{SL}-\mathrm{MoS}_{2} / \mathrm{ZnO}$ NPs are $2184 \mu \mathrm{mol}$ $\mathrm{g}^{-1} \mathrm{~h}^{-1}$ and $1152 \mu \mathrm{mol} \mathrm{g}^{-1} \mathrm{~h}^{-1}$, respectively. On the other hand, the combination with their non-polar counterparts were also tested which still shows no improvement of the photocatalytic activities of overall water splitting reaction (Fig.S16). It is noted that there has been nearly 4 times promotion in activity when blended with polar $\mathrm{CeO}_{2} \mathrm{NCs}$ support. Apparently, the greatly prolonged exciton lifetimes result in the remarkable enhancement of photocatalytic activities. The correlation of exciton lifetimes derived from TRPL results with photocatalytic water splitting activity is also summarised in Table 1 for comparison, more details can also be found in the Table S1 and S2.
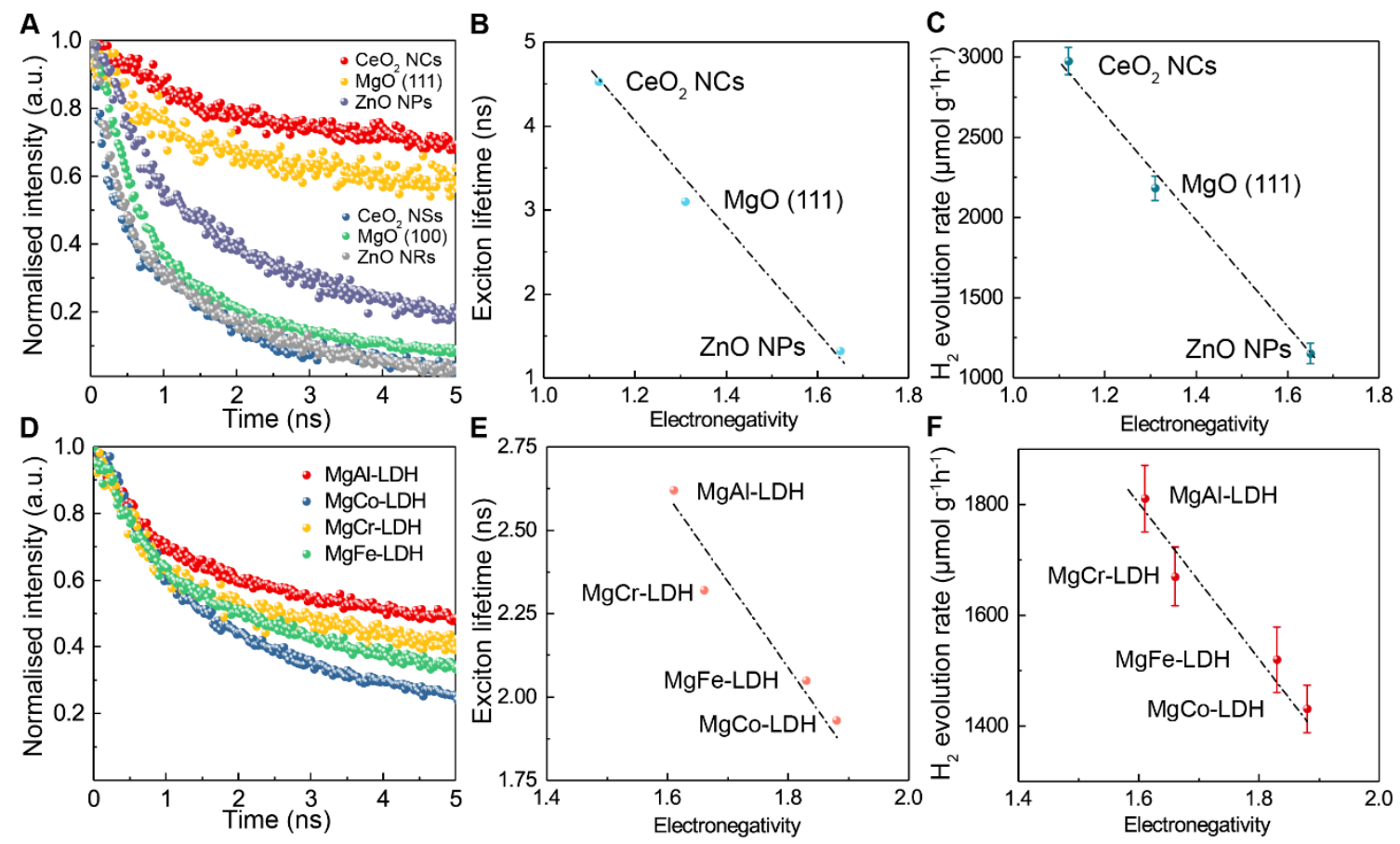
Figure 2. The relationship of local polarity between photocatalytic performance and exciton lifetime. (A) Comparison of the TRPL spectra of Ru:SL-MoS 2 after mixed with polar and non-polar faceted oxide supports; (B, E) Exciton lifetimes of the Ru:SL-MoS 2 photocatalysts promoted by polar-faceted oxide supports and LDHs, plotted against the electronegativities. The errors were shown in the Table 1 and Table S2; (C, F) Photocatalytic activities of Ru:SL-MoS 2 after combined with polar-faceted oxides and LDHs, respectively, plotted against the electronegativities. Error bars are given as the standard deviations; (D) TRPL spectra of Ru:SL-MoS 2 after combined with LDHs,

Table 1. The correlation of exciton lifetime and photocatalytic water splitting activity of the photocatalysts studied in this work.

\begin{tabular}{|llll|}
\hline Entry & Photocatalyst & Exciton lifetime (ns) & Activity $\left(\boldsymbol{\mu m o l ~ h}^{\mathbf{- 1}} \mathbf{~ g}^{-\mathbf{1}}\right)$ \\
\hline 1 & $\mathrm{Ru}: \mathrm{SL}-\mathrm{MoS}_{2}$ & $1.02 \pm 0.01$ & $821 \pm 33$ \\
\hline 2 & $\mathrm{Ru}: \mathrm{SL}-\mathrm{MoS}_{2} / \mathrm{CeO}_{2} \mathrm{NCs}$ & $4.53 \pm 0.05$ & $2977 \pm 85$ \\
\hline 3 & $\mathrm{Ru}: \mathrm{SL}_{-\mathrm{MoS}_{2} / \mathrm{CeO}_{2} \mathrm{NSs}}$ & $1.04 \pm 0.03$ & $770 \pm 31$ \\
\hline 4 & $\mathrm{Ru}: \mathrm{SL}_{-\mathrm{MoS}_{2} / \mathrm{MgO}(111)}$ & $3.10 \pm 0.02$ & $2184 \pm 76$ \\
\hline 5 & $\mathrm{Ru}: \mathrm{SL}-\mathrm{MoS}_{2} / \mathrm{MgO}(100)$ & $1.02 \pm 0.04$ & $808 \pm 40$ \\
\hline 6 & $\mathrm{Ru}: \mathrm{SL}-\mathrm{MoS}_{2} / \mathrm{ZnO} \mathrm{NPs}$ & $1.32 \pm 0.03$ & $1152 \pm 65$ \\
\hline 7 & $\mathrm{Ru}: \mathrm{SL}-\mathrm{MoS}_{2} / \mathrm{ZnO} \mathrm{NRs}$ & $1.03 \pm 0.02$ & $839 \pm 29$ \\
\hline 8 & $\mathrm{Ru}: \mathrm{SL}-\mathrm{MoS}_{2} / \mathrm{MgAl}-\mathrm{LDH}$ & $2.62 \pm 0.05$ & $1811 \pm 60$ \\
\hline 9 & $\mathrm{Ru}: \mathrm{SL}-\mathrm{MoS}_{2} / \mathrm{MgCo}-\mathrm{LDH}$ & $1.93 \pm 0.03$ & $1431 \pm 43$ \\
\hline 10 & $\mathrm{Ru}: \mathrm{SL}-\mathrm{MoS}_{2} / \mathrm{MgCr}-\mathrm{LDH}$ & $2.32 \pm 0.04$ & $1670 \pm 53$ \\
\hline 11 & $\mathrm{Ru}: \mathrm{SL}-\mathrm{MoS}_{2} / \mathrm{MgFe}-\mathrm{LDH}$ & $2.05 \pm 0.02$ & $1520 \pm 59$ \\
\hline
\end{tabular}

To strike more understanding of the controlling of the charge separation process via the polarisation effect by using polar-faceted oxides, we tried to find the relationship between the polarity and photocatalytic activities. Obviously, polarity is related to the electronegativity difference of the metal and oxygen in a polarfaceted oxide, and the metal with lower electronegativity gives higher polarity near the surface of the polarfaceted oxide in this negative to positive layer configuration. Thus, the photocatalytic activities and exciton lifetimes are plotted against the polarity, respectively (Fig. 2B and Fig. 2C). It is interesting to see that both the photocatalytic activities and exciton lifetimes show apparent linear relationships with the polarity within experimental errors, which confirm that the exciton lifetime of a photocatalyst can be proportionally prolonged by the local electric field introduced by the polarisation effect of the polar-faceted oxide supports. Bearing in mind that the local polarisation effect observed so far should not be limited to the polar-faceted metal oxides, this technique may be extended to other kinds of solid materials. As a result, further exploration of the local polarisation effect was attempted on using layered double hydroxides (LDHs) structure. As is well known, LDHs are well-defined materials with layered structures, which consist of metal layers and 
hydroxide layers carrying alternative negative and positive charge along their main crystallographic [100] direction. Thus, the LDHs can also introduce a local electric field via the surface polarity. Moreover, the surface polarity can be more flexibly controlled by changing the metal elements and ratios of the LDHs. Various LDHs were thus prepared by co-precipitation method, including MgAl-LDH, MgCr-LDH, MgFeLDH and MgCo-LDH, and each of them was assembled with the Ru:SL-MoS 2 by refluxing in water. As shown in Fig. S17, the structure of each LDH is confirmed by XRD with their characteristic peaks observed at $2 \theta=11.4^{\circ}$ and $22.9^{\circ}$, implying the existence of (003) and (006) facets of the LDHs [30,31].

The recombination process of the photo-excited electron-hole pairs of the as-prepared materials was subsequently investigated by TRPL, as is summarised in Fig. 2D. Not surprisingly, the charge carrier separation is substantially facilitated when Ru:SL-MoS 2 is mixed with LDHs: the exciton lifetime increases from $1.02 \mathrm{~ns}$ of $\mathrm{Ru}: \mathrm{SL}-\mathrm{MoS}_{2}$ to $2.62 \mathrm{~ns}$ of $\mathrm{Ru}: \mathrm{SL}-\mathrm{MoS}_{2} / \mathrm{MgAl}-\mathrm{LDH}$. Photocatalytic activity of water splitting reaction was evaluated. As expected, the photocatalytic performance was also apparently enhanced compared with Ru:SL-MoS 2 . It is exciting to see that the most common MgAl-LDH shows the highest enhancement factor among the four selected LDHs, leading to a hydrogen evolution rate of $1811 \mu^{\mathrm{mol} \mathrm{g}^{-1}} \mathrm{~h}$ 1. This value is among the best of the visible-light-driven overall water splitting systems. The layered structure of LDHs allows the formation of the oriented polarisation inside the LDHs, which leads to the presence of the LEF near the surface, therefore prolongs the exciton lifetime of Ru:SL-MoS 2 , resulting in the enhanced photocatalytic activities. Considering that all these LDHs share similar structures according to the XRD results with only the trivalent metal altered, it is reasonable to argue that the polarisation intensities near the surface are proportional to the electronegativities of the trivalent metals. Thus, their exciton lifetimes and photocatalytic activities are then plotted against the electronegativities of the trivalent metals. Obviously, both exciton lifetime and photocatalytic activity follow a similar nice linear trend against the electronegativity, akin to the same condition as the polar-faceted oxides (Fig. 2E and Fig. 2F). 

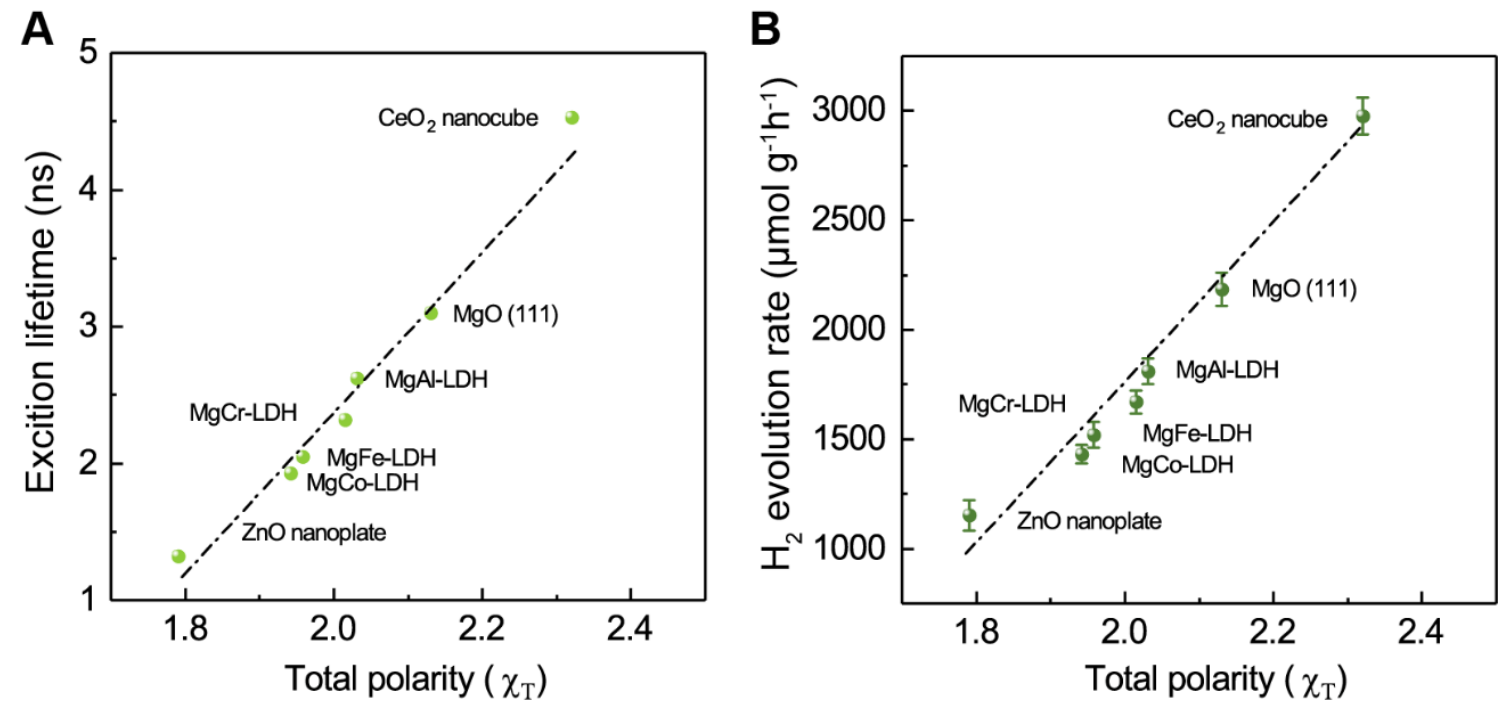

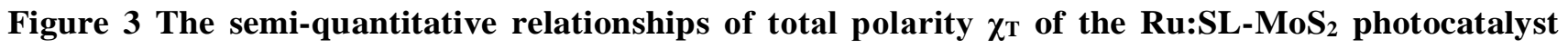
enhanced by polar-faceted metal oxides and LDHs supports (A) the relationship between total polarity $\chi_{\mathrm{T}}$ and exciton lifetimes; (B) the relationship between total polarity $\chi_{\mathrm{T}}$ and photocatalytic hydrogen evolution rates. Error bars are given as the standard deviations.

Although the polar-faceted metal oxides and LDHs as supports have been studied separately, further rationalisation of these photocatalyst supports with prolonged exciton lifetimes may be useful for the generalisation of the correlation. It should be borne in mind that the binary metal oxides and LDHs have very different compositions and structures. Thus, the contributions of $\mathrm{Mg}$ and trivalent metals are taken into account with considering their metal ratios. So, a total polarity is calculated, as shown in the following equations:

For polar-faceted metal oxides:

$$
\text { Total polarity } \chi_{\mathrm{T}}=\chi_{\mathrm{oxygen}}-\chi_{\text {metal }}
$$

For LDHs:

$$
\text { Total polarity } \chi_{\mathrm{T}}=\chi_{\mathrm{oxygen}}-\left(\chi_{\mathrm{Mg}} \times \omega_{\mathrm{Mg}}+\chi_{2} \times \omega_{2}\right)
$$

( $\chi_{\text {oxygen }}, \chi_{\text {metal }}, \chi_{\mathrm{Mg}}, \chi_{2}$ - The electronegativity of oxygen, metal ions in metal oxides, magnesium and the trivalent metal ions in the LDHs, $\omega_{\mathrm{Mg}}, \omega_{2}-$ The molar percentage of magnesium and the trivalent metal in the LDHs.) 

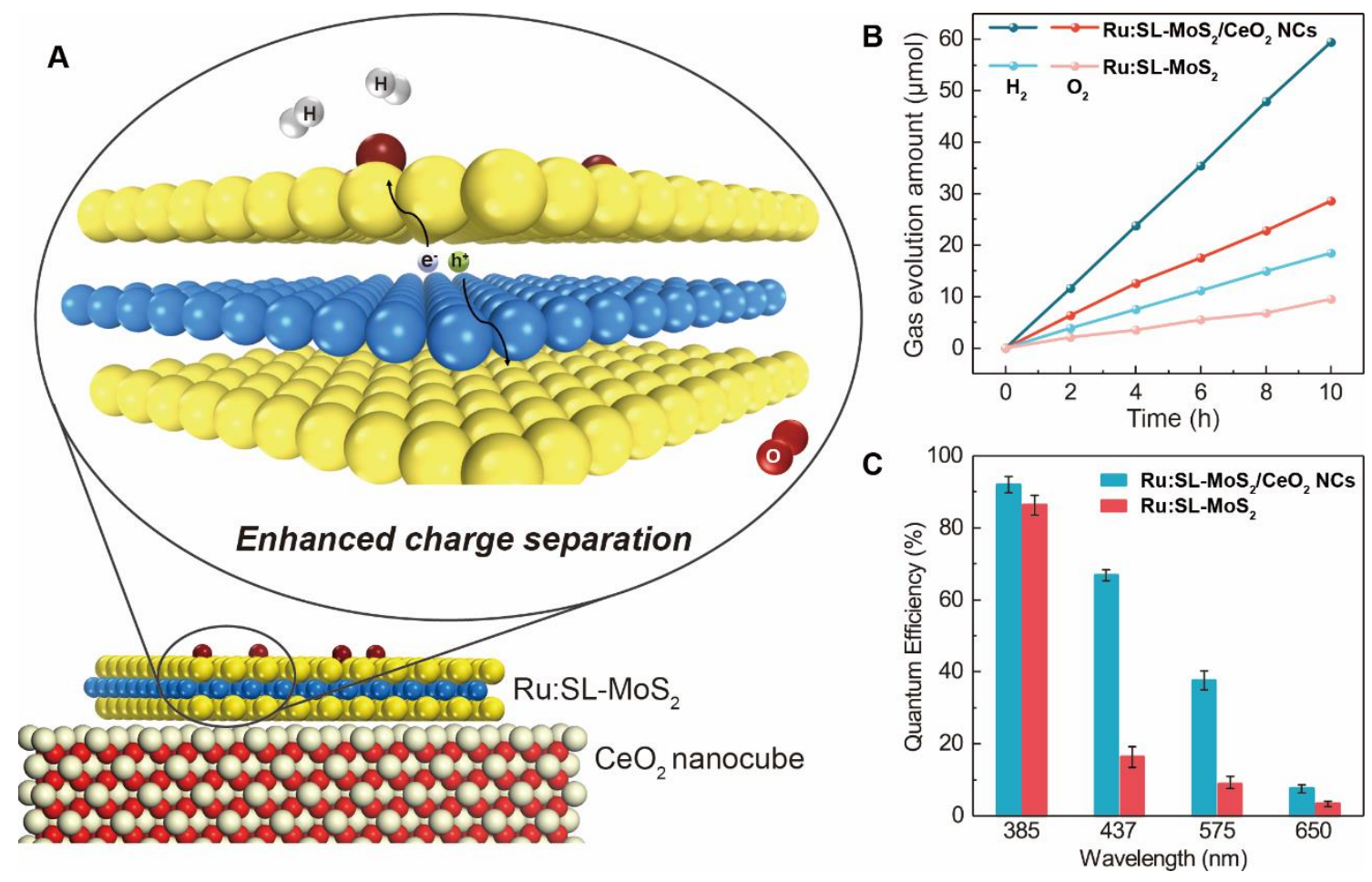

Figure 4 Photocatalytic water splitting performance tests. (A) Schematic illustration of the charge separation process promoted by local polarisation introduced by polar-faceted oxide, resulting in enhanced photocatalytic water splitting performance (yellow: sulphur; blue: molybdenum; brown: ruthenium; ivory: cerium; red: oxygen); (B) Photocatalytic activity tests: stable stoichiometric decomposition of water to 2:1 $\mathrm{H}_{2} / \mathrm{O}_{2}$ at $270^{\circ} \mathrm{C}$ in an autoclave with no sacrificial reagent over Ru:SL-MoS 2 with and without polar $\mathrm{CeO}_{2}$ NCs support at constant rate for 10 hours; (C) QE of Ru:SL-MoS 2 with and without polar $\mathrm{CeO}_{2} \mathrm{NCs}$ using incident light with wavelengths of $385 \mathrm{~nm}, 437 \mathrm{~nm}, 575 \mathrm{~nm}$ and $650 \mathrm{~nm}$, respectively.

In the Equation (1), the total polarity $\chi_{\mathrm{T}}$ of polar-faceted oxides is defined by the difference between the electronegativity of oxygen and metal; while for those of LDHs, both trivalent metal and bivalent metal $(\mathrm{Mg})$ are considered, and the contribution of each is normalised depending on the molar ratio, as demonstrated in Equation (2). Then all the photocatalytic activities and exciton lifetimes of the Ru:SL-MoS 2 based materials are plotted against the total polarity. Interestingly, new linear relationships are clearly observed, as a result, exciton lifetimes are found proportional to the total polarities $\chi_{\mathrm{T}}$ (Fig. 3A), which further confirms that the charge separation process can be controlled by tuning the local polarity through different polar-faceted supports. Such facilitated charge separation therefore contributes to the enhanced photocatalytic performance, making the photocatalytic water splitting activities also proportional to the total polarities $\chi_{\mathrm{T}}$ (Fig. 3B). Strong LEF is exerted to the catalyst by the surface of the polar-faceted materials, and such polarisation effect prolong the exciton lifetimes at the interfaces, which largely enhances the photocatalytic 
activities. This LEF promoted mechanism is also demonstrated in the Fig. 4A. According to this study, it is found that the local polarisation effect introduced by polar-faceted supports plays a very important role in the photocatalytic water splitting performance. The polar-faceted metal oxides consist of alternate $\mathrm{O}$ and metal ions layers, terminated with either negative charged O-layer or positive charged metal-layer, respectively. The polarisation on the surface arises from the net non-zero dipole moment of such ionic arrangements, which is defined by both the charge densities and distances between these layers $(\mu=\sigma \mathrm{R}$, where $\mu, \sigma$ and $\mathrm{R}$ represents the dipole moment, charge density and interlayer distance, respectively) [32,33]. For binary compounds like metal oxides with M-O without considering charge numbers for cation and anion and their structural factor, the strength of polarisation can simply correlate to the difference in electronegativities (EN) of the M-O constituents. Therefore, in this work as shown in Fig. 3, such method was applied and the strength of polarity on the surface was calculated as the electronegativity differences between metal cations and $\mathrm{O}$ anions. However, $\mathrm{Ce}$ has higher cationic charge number than the second best $\mathrm{Mg}$ and thus giving much higher charge density hence the higher polarisation. Excitingly, the photocatalytic performance appears to show a strong dependency on the change of polarity strength, with the exciton lifetime showing a similar trend. As a result, taking the charge numbers and EN difference into account, $\mathrm{CeO}_{2}$ nanocubes with polar (100) exposed facets shows the highest surface polarity, thus gives the strongest local polarisation effect to the Ru:SL-MoS photocatalyst. The high polarisation of this support can also be reflected by the probe-assisted solid-state NMR and TRPL results as shown in Fig. S11 and Fig. 3.

Ru:SL-MoS 2 and Ru:SL-MoS $/ \mathrm{CeO}_{2}$ NCs were then tested for a period of 10 hours, both of which showed good stability without obvious change of the hydrogen evolution rates. As shown in Fig. 4B, both catalysts show a stable production of $\mathrm{H}_{2}$ and $\mathrm{O}_{2}$ stoichiometrically in a 2:1 ratio. It is widely agreed that for a photocatalytic system, the QE, which evaluates the conversion efficiency of the photons at a certain wavelength to hydrogen, is one of the most important parameters when evaluating the performance of renewable solar energy to hydrogen fuel systems [34]. Different from the overall solar-to-hydrogen conversion efficiency or hydrogen evolution rate, the QE provides more detailed and fundamental information about the photocatalytic systems, bearing in mind that a photocatalyst performs differently when harness the photons from different wavelengths. Remarkable QEs are frequently reported in the presence of sacrificial reagents, unfortunately, for the overall water splitting system. Almost all the reported QEs are extremely low under the visible light irradiation (rarely exceeding $5 \%$ at $420 \mathrm{~nm}$ ), which cannot reach the requirement of any practical applications. In our photocatalytic system, the $\mathrm{QE}$ is evaluated at $270{ }^{\circ} \mathrm{C}$ with the highest water ionisation at wavelengths ranging from ultraviolet to visible light using different band pass filters (Fig. S18). As illustrated in Fig. 4C, high QE of about 90\% are obtained for each photocatalyst at 385 $\mathrm{nm}$ due to high absorption. It is exciting that when the light irradiation is changed to visible light, an extraordinary QE of $66.8 \%$ for Ru:SL- $\mathrm{MoS}_{2} / \mathrm{CeO}_{2} \mathrm{NCs}$ can still be maintained at $437 \mathrm{~nm}$, which is more 
than 10 times higher than most results reported so far. QE sharply drops with further increasing the wavelength due to the rapid decrease of photon absorption at longer wavelengths [14]. However, it is surprising that $\mathrm{MoS}_{2}$-based photocatalysts show considerable $\mathrm{QE}$ of $7.5 \%$ at even $650 \mathrm{~nm}$ at elevated temperature and more detailed comparison of the activities and QE can be found in Tables S3 and S4. An example of the QE calculation is shown in the Supplementary Discussion.

So far, the local polarisation effects introduced by the polar-faceted support materials have been systematic studied and exciting photocatalytic water splitting performance can be achieved over SL-MoS 2 based materials. The feasibility of using solely the renewable solar energy to supply both the required thermal heat and visible photons for photocatalytic splitting of water for $\mathrm{H}_{2} / \mathrm{O}_{2}$ production are also considered. To demonstrate this point, a four-mirror floating-zone light furnace equipped with four halogen lamps and concave mirrors was used to mimic a solar light concentrator, in which not any other direct thermal energy input from an electrical device was used, and the reactor temperature of $270{ }^{\circ} \mathrm{C}$ was maintained by the light source with the black body radiation [20]. Constant $\mathrm{H}_{2}$ evolution rates of about $3 \mathrm{mmol} \mathrm{g}^{-1} \mathrm{~h}^{-1}$ and $10 \mathrm{mmol}$ $\mathrm{g}^{-1} \mathrm{~h}^{-1}$ are achieved over Ru:SL-MoS 2 without and with polar $\mathrm{CeO}_{2}$ support (see Fig. S19), respectively, indicating the great potential of further application of this photocatalytic water splitting system over nanocatalysts.

\section{Conclusion}

In conclusion, a series of SL-MoS 2 based photocatalysts are studied in this work, which show promising catalytic performance toward water splitting reaction at elevated temperature under visible light illumination. It is demonstrated that the photo-generated charge carrier separation can be substantially facilitated by assembling to polar-faceted materials as supports, which can exert strong LEF in proximity of the interfaces. The resulted prolonged exciton lifetimes allow the charge carriers travel to the surface of the photocatalysts and make the subsequent chemical reactions take place. This local polarisation effect on excitons is systematic explored and studied with the help of TRPL, which confirms the enhancement of the exciton lifetime and the control of the charge separation process. It is noted that both photocatalytic activities and exciton lifetimes show an obvious linear relationship with the intensity of the LEF. Therefore, the local polarisation effect can give a strong enhancement to photocatalysis by promoting the separation of the photoexcited electron-hole pairs. This observation provides a simple but versatile means to control the recombination process, leading to rational design of the photocatalysts using polar support materials, not only for water splitting system but may also be valuable for other photocatalytic processes, which may also contribute to the future utilisation of solar energy. 


\section{Experimental section}

\subsection{Materials}

$\mathrm{MoS}_{2}$ powder (reagent grade, Sigma-Aldrich); Cerium nitrate hexahydrate (reagent grade, Sigma-Aldrich); Magnesium chloride (reagent grade, Sigma-Aldrich); Benzoic acid (reagent grade, Sigma-Aldrich); Zinc acetate dihydrate (reagent grade, Sigma-Aldrich); 1,2-ethanediamine (reagent grade, Sigma-Aldrich); Iron chloride hexahydrate (reagent grade, Alfa Aesar); n-butyllithium/hexane (reagent grade, Sigma-Aldrich); Polyvinylpyrrolidone (PVP, reagent grade, Sigma-Aldrich); Chromium(III) nitrate nonahydrate (reagent grade, Sigma-Aldrich); Manganese chloride tetrahydrate (reagent grade, Sigma-Aldrich); Ruthenium(III) acetylacetonate (reagent grade, Sigma-Aldrich); Nickel chloride hexahydrate (reagent grade, Sigma-Aldrich); Cobalt nitrate hexahydrate (reagent grade, Sigma-Aldrich); Hydrogen tetrachloroaurate trihydrate (reagent grade, Sigma-Aldrich); Isopropanol (99.9\%, Sigma-Aldrich); Methanol (anhydrous, $\geq 99.8 \%$ (HPLC), Sigma-Aldrich); $\mathrm{H}_{2} \mathrm{SO}_{4}$ ( $\geq 98 \%$, Sigma-Aldrich); Ammonia gas (anhydrous, BOC); Argon gas (99.99\%, BOC); Helium gas (99.99\%, BOC); Nitrogen gas $(99.99 \%$, BOC).

\subsection{Methods}

Synthesis of single layer $\mathrm{MoS}_{2}$ and metal doping. Single layer $\mathrm{MoS}_{2}$ was prepared based on our previous report [10]: $2 \mathrm{~g}$ of commercial bulk $\mathrm{MoS}_{2}$ powder was soaked in $16 \mathrm{~mL}$ of $1.6 \mathrm{M} \mathrm{n}$-butyllithium/hexane for $48 \mathrm{~h}$ in $\mathrm{N}_{2}$ atmosphere. After the lithium intercalation process, the obtained intercalated $\mathrm{MoS}_{2}$ was washed with $50 \mathrm{~mL}$ of hexane to remove excess butyl lithium and organics and centrifuged and then dried under $\mathrm{N}_{2}$. Then the powder was immersed in $500 \mathrm{~mL}$ of water and the resulting suspension was sonicated to assist the exfoliation process. The dispersion was centrifuged at 5,000 $\mathrm{rpm}$ for $15 \mathrm{~min}$ to remove unexfoliated precursors and only the supernatant was collected. Eventually, the exfoliated $\mathrm{MoS}_{2}$ single layers remained totally suspended in aqueous solution. To collect the $\mathrm{SL}-\mathrm{MoS}_{2}$ powder, $\mathrm{HCl}$ solution was added dropwise into the above colloid until the $\mathrm{pH}$ value reaching around 7 . The precipitate was then washed and collected by centrifugation several times and the final product was dried under vacuum overnight.

Metal doped SL-MoS 2 photocatalysts (denoted as M:SL-MoS 2 , M represents the metal doping) were prepared using a hydrothermal method. Certain amount of corresponding metal precursors (2 wt.\% calculated loading amount) and $150 \mathrm{mg}$ of thiourea were added to $10 \mathrm{ml}$ of water and left overnight to form the complex. Subsequently this solution was then added into $50 \mathrm{~mL}$ colloid containing $180 \mathrm{mg} \mathrm{SL-MoS}$ (30 $\mathrm{v} / \mathrm{v} \%$ isopropanol/water with $50 \mathrm{mg}$ of polyvinylpyrrolidone). A mixed homogeneous solution was then transferred to an autoclave, followed by hydrothermal treatment at $160{ }^{\circ} \mathrm{C}$ for $24 \mathrm{~h}$. After the reaction, the 
precipitate was washed three times using deionised water and then dried under vacuum for $12 \mathrm{~h}$ and finally treated with $\mathrm{H} 2$ at $300{ }^{\circ} \mathrm{C}$ for $1 \mathrm{~h}$ prior to storage.

Assembly of the photocatalysts with metal oxides or LDHs. Ru:SL-MoS ${ }_{2}$ was mixed and grinded with different metal oxides thoroughly at 50:50 wt.\% and allowed to disperse in water and sonicated for 2 hours, filtered, dried and calcined in $\mathrm{N}_{2}$ at $400{ }^{\circ} \mathrm{C}$ for $2 \mathrm{~h}$ prior to use. The assembly of photocatalysts with LDHs was modified from a method reported in literature [14]: Ru:SL-MoS ${ }_{2}$ was mixed with different LDHs thoroughly at 50:50 wt. $\%$ and dispersed in $50 \mathrm{~mL}$ water, followed by refluxing at $60{ }^{\circ} \mathrm{C}$ for $1 \mathrm{~h}$. Subsequently the flask was allowed to cool to room temperature naturally and then the solid was separated by centrifugation at $5000 \mathrm{rpm}$ for $10 \mathrm{~min}$ and washed with DI water for 3 times. The product was dried under vacuum for further use.

\subsection{Characterisations}

The morphologies of the materials were studied by High-angle annular dark field scanning transition electron microscopy (HAADF-STEM, JEOL-JEM2100 Aberration-Corrected Transmission Electron Microscope). XRD measurements were performed on a Bruker D8 Advance diffractometer with LynxEye detector and Cu K $\alpha 1$ radiation $(\lambda=1.5406 \AA)$. Continuous-wave EPR spectra were obtained by using an X-band $(9.4 \mathrm{GHz})$ Bruker EMX EPR spectrometer. The exciton lifetimes were evaluated by time-resolved photoluminescence (TRPL) spectroscopy using a bespoke micro photoluminescence setup in which a Ti-Sapphire laser $(\lambda=266$ $\mathrm{nm}$, pulse duration $=150 \mathrm{fs}$, repetition rate $=76 \mathrm{MHz}$ ) is directed onto the sample. The exciton lifetime is obtained by fitting corresponding background-corrected PL spectrum.

\subsection{Evaluation of photocatalytic performances}

Photocatalytic water splitting activity tests. The photocatalytic activity was determined by measuring the amount of hydrogen and oxygen evolved from the water splitting. Reactions were carried out in a close 25 $\mathrm{mL}$ stainless steel autoclave system equipped with two quartz windows $(10 \mathrm{~mm}$ in diameter and $18 \mathrm{~mm}$ in thickness). In a typical experiment, catalyst contains $2 \mathrm{mg}$ of SL- $\mathrm{MoS}_{2}$ is added to $10 \mathrm{~mL}$ Milli-Q $\mathrm{H}_{2} \mathrm{O}$ in a glass lining $(20 \mathrm{~mm}$ i.d. $\times 24 \mathrm{~mm}$ o.d. $\times 52 \mathrm{~mm}$ height) under vigorous magnetic stirring $(750 \mathrm{rpm})$, then the autoclave was pressurised with 2 bar of Ar gas as inert gas after being well sealed. The mixture in the reactor would then be allowed to heat up to the designated temperature $\left(270{ }^{\circ} \mathrm{C}\right)$.

Tungsten light (70 W, Glamox Professional 2000) was then applied through the quartz windows to provide visible light irradiation after the autoclave reached certain temperature. After $2 \mathrm{~h}$ reaction, the autoclave was cooled down naturally to room temperature and the amounts of hydrogen and oxygen were measured by gas 
chromatography (GC) equipped with thermoconductivity detectors (TCD) with $\mathrm{He}$ and $\mathrm{N}_{2}$ as carrier gas, respectively.

Quantum efficiency (QE) measurements and calculation. Apparent quantum efficiency was measured in the same autoclave and conditions were kept the same as those for a typical photocatalytic test, while the autoclave was then irradiated by a $300 \mathrm{~W}$ Xenon lamp (Newport) using bandpass filters of $385 \pm 40 \mathrm{~nm}$, $437 \pm 10 \mathrm{~nm}, 575 \pm 20 \mathrm{~nm}$ and $650 \pm 20 \mathrm{~nm}$, respectively (as shown in Fig. S18). Numbers of photons were calculated from the irradiation powers in each wavelength region measured by a light meter at the corresponding wavelengths. The apparent quantum efficiency can be calculated using the following equation [35], and a typical calculation of QE is given in the Supplementary Discussion:

$\mathrm{QE}(\%)=($ Number of evolved hydrogen molecules $\times 2) /($ Number of incident photons $) \times 100 \%$

\section{References}

[1] F.M. Pesci, M.S. Sokolikova, C. Grotta, P.C. Sherrell, F. Reale, K. Sharda, N. Ni, P. Palczynski, C. Mattevi, ACS Catal. 7 (2017) 4990-4998.

[2] T. Jia, A. Kolpin, C. Ma, R. Chau-Ting Chan, W.M. Kwok, S.C.E. Tsang, Chem. Commun. 50 (2014) 1185-1188.

[3] X.L. Yin, L.L. Li, W.J. Jiang, Y. Zhang, X. Zhang, L.J. Wan, J.S. Hu, ACS Appl. Mater. Interfaces 8 (2016) 15258-15266.

[4] D.P. Kumar, S. Hong, D.A. Reddy, T.K. Kim, J. Mater. Chem. A 4 (2016) 18551-18558.

[5] K. Chang, M. Li, T. Wang, S. Ouyang, P. Li, L. Liu, J. Ye, Adv. Energy Mater. 5 (2015) 1402279.

[6] B. Sun, Z. Liang, Y. Qian, X. Xu, Y. Han, J. Tian, ACS Appl. Mater. Interfaces 12 (2020) 72577269 .

[7] J. Zhang, X. Tian, M. Liu, H. Guo, J. Zhou, Q. Fang, Z. Liu, Q. Wu, J. Lou, J. Am. Chem. Soc. 141 (2019) 19269-19275.

[8] M. Chhowalla, H.S. Shin, G. Eda, L.J. Li, K.P. Loh, H. Zhang, Nat. Chem. 5 (2013) 263-275.

[9] T.H.M. Lau, X. Lu, J. Kulhavý, S. Wu, L. Lu, T.S. Wu, R. Kato, J.S. Foord, Y.L. Soo, K. Suenaga, S.C.E. Tsang, Chem. Sci. 9 (2018) 4769-4776.

[10] G. Liu, A.W. Robertson, M.M.J. Li, W.C.H. Kuo, M.T. Darby, M.H. Muhieddine, Y.C. Lin, K. Suenaga, M. Stamatakis, J.H. Warner, S.C.E. Tsang, Nat. Chem. 9 (2017) 810-816.

[11] K.F. Mak, C. Lee, J. Hone, J. Shan, T.F. Heinz, Phys. Rev. Lett. 105 (2010) 2-5.

[12] X. Zong, H. Yan, G. Wu, G. Ma, F. Wen, L. Wang, C. Li, J. Am. Chem. Soc. 130 (2008) 71767177.

[13] L. Li, J. Yan, T. Wang, Z.J. Zhao, J. Zhang, J. Gong, N. Guan, Nat. Commun. 6 (2015) 5881.

[14] J. Li, G. Zhan, Y. Yu, L. Zhang, Nat. Commun. 7 (2016) 11480.

[15] J. Li, L. Cai, J. Shang, Y. Yu, L. Zhang, Adv. Mater. 28 (2016) 4059-4064.

[16] F. Chen, H. Huang, L. Guo, Y. Zhang, T. Ma, Angew. Chem. Int. Ed. 58 (2019) 10061-10073.

[17] W. Hu, L. Lin, R. Zhang, C. Yang, J. Yang, J. Am. Chem. Soc. 139 (2017) 15429-15436. 
[18] Z. Zhang, Q. Qian, B. Li, K.J. Chen, ACS Appl. Mater. Interfaces 10 (2018) 17419-17426.

[19] M.A. Lukowski, A.S. Daniel, F. Meng, A. Forticaux, L. Li, S. Jin, J. Am. Chem. Soc. 135 (2013) 10274-10277.

[20] Y. Li, Y.-K. Peng, L. Hu, J. Zheng, D. Prabhakaran, S. Wu, T.J. Puchtler, M. Li, K.-Y. Wong, R.A. Taylor, S.C.E. Tsang, Nat. Commun. 10 (2019) 4421.

[21] Y. Li, S.C.E. Tsang, Mater. Today Sustain. 9 (2020) 100032.

[22] W. Qiao, S. Yan, X. Song, X. Zhang, X. He, W. Zhong, Y. Du, Appl. Surf. Sci. 359 (2015) 130136.

[23] S. Yan, W. Qiao, X. He, X. Guo, L. Xi, W. Zhong, Y. Du, Appl. Phys. Lett. 106 (2015) 012408.

[24] P. Rivera, J.R. Schaibley, A.M. Jones, J.S. Ross, S. Wu, G. Aivazian, P. Klement, K. Seyler, G. Clark, N.J. Ghimire, J. Yan, D.G. Mandrus, W. Yao, X. Xu, Nat. Commun. 6 (2015) 6242.

[25] J. Mahmood, F. Li, S.M. Jung, M.S. Okyay, I. Ahmad, S.J. Kim, N. Park, H.Y. Jeong, J.B. Baek, Nat. Nanotechnol. 12 (2017) 441-446.

[26] Y.K. Peng, L. Ye, J. Qu, L. Zhang, Y. Fu, I.F. Teixeira, I.J. McPherson, H. He, S.C.E. Tsang, J. Am. Chem. Soc. 138 (2016) 2225-2234.

[27] L. Ye, A.H. Mahadi, C. Saengruengrit, J. Qu, F. Xu, S.M. Fairclough, N. Young, P.L. Ho, J. Shan, L. Nguyen, F.F. Tao, K. Tedsree, S.C.E. Tsang, ACS Catal. 9 (2019) 5171-5177.

[28] Y.K. Peng, Y. Fu, L. Zhang, I.F. Teixeira, L. Ye, H. He, S.C.E. Tsang, ChemCatChem 9 (2017) $155-160$.

[29] Y.K. Peng, Y. Hu, H.L. Chou, Y. Fu, I.F. Teixeira, L. Zhang, H. He, S.C.E. Tsang, Nat. Commun. 8 (2017) 675.

[30] J. Zhang, X. Xie, C. Li, H. Wang, L. Wang, RSC Adv. 5 (2015) 29757-29765.

[31] C.G. Silva, Y. Bouizi, V. Fornés, H. García, J. Am. Chem. Soc. 131 (2009) 13833-13839.

[32] C. Noguera, J. Phys. Condens. Matter 12 (2000) R367-R410.

[33] D. Vanderbilt, R.D. King-Smith, Phys. Rev. B 48 (1993) 4442-4455.

[34] J. Wang, D.N. Tafen, J.P. Lewis, Z. Hong, A. Manivannan, M. Zhi, M. Li, N. Wu, J. Am. Chem. Soc. 131 (2009) 12290-12297.

[35] J. Liu, Y. Liu, N. Liu, Y. Han, X. Zhang, H. Huang, Y. Lifshitz, S.T. Lee, J. Zhong, Z. Kang, Science 347 (2015) 970-974.

\section{Acknowledgements}

The support for this project from the EPSRC in the UK (grant EP/K040375/1) is gratefully acknowledged. The authors also wish to thank Dr Chen Wu and Mr Zihan Wang of Zhejiang University, China for their help in some TEM and EDX analyses.

\section{Author contributions}


Y.L., S.W., J.Z., and Y.-K.P. prepared, characterised and tested the catalysts. Y.L. and D.P. performed the light furnace experiments. Y.L. and R.A.T. collected and analysed the TRPL data. Y.L. and S.C.E.T. wrote the paper in discussion with R.A.T. S.C.E.T. supervised the overall project.

\section{Competing interests}

The authors declare no competing interests.

\section{Additional information}

Supplementary information is available for this paper. 